\title{
TRACER: a resource to study the regulatory architecture of the mouse genome
}

\author{
Chao-Kung Chen ${ }^{1}$, Orsolya Symmons ${ }^{2}$, Veli Vural Uslu², Taro Tsujimura ${ }^{2}$, Sandra Ruf ${ }^{2}$, Damian Smedley ${ }^{1,3^{*}}$ \\ and François Spitz ${ }^{2^{*}}$
}

\begin{abstract}
Background: Mammalian genes are regulated through the action of multiple regulatory elements, often distributed across large regions. The mechanisms that control the integration of these diverse inputs into specific gene expression patterns are still poorly understood. New approaches enabling the dissection of these mechanisms in vivo are needed.

Results: Here, we describe TRACER (http://tracerdatabase.embl.de), a resource that centralizes information from a large on-going functional exploration of the mouse genome with different transposon-associated regulatory sensors. Hundreds of insertions have been mapped to specific genomic positions, and their corresponding regulatory potential has been documented by analysis of the expression of the reporter sensor gene in mouse embryos. The data can be easily accessed and provides information on the regulatory activities present in a large number of genomic regions, notably in gene-poor intervals that have been associated with human diseases.

Conclusions: TRACER data enables comparisons with the expression pattern of neighbouring genes, activity of surrounding regulatory elements or with other genomic features, revealing the underlying regulatory architecture of these loci. TRACER mouse lines can also be requested for in vivo transposition and chromosomal engineering, to analyse further regions of interest.
\end{abstract}

Keywords: Gene regulation and expression, Genome organisation, Regulatory landscapes, Chromosomal engineering, Mouse models of human structural variation

\section{Background}

Genes occupy only a small fraction of mammalian genomes. Accordingly, intergenic regions can extend up to several megabases, and the functional importance of these regions is being growingly recognized [1] (Figure 1). Notably, these regions comprise important elements that control gene expression [3]. Enhancer elements are frequently found hundreds of kilobases away from the promoter of the gene they control, sometimes even separated from it by unrelated genes [4-11]. These remote enhancers can be essential for gene expression, as shown by human disorders resulting from their mutation or disruption by chromosomal rearrangements [12-16]. The

\footnotetext{
*Correspondence: damian@ebi.ac.uk; spitz@embl.de

${ }^{1}$ European Bioinformatics Institute - European Molecular Biology Laboratory, Wellcome Trust Genome Campus, Hinxton, Cambridge CB10 1SD, UK ${ }^{2}$ Developmental Biology Unit - European Molecular Biology Laboratory, Heidelberg, Germany

Full list of author information is available at the end of the article
}

importance of these intergenic regions in human phenotypic diversity and disease susceptibility is further emphasized by the significant proportion of risk alleles that have been identified in gene-desert intervals [3,17-20]. Thus, there is a pressing need to better characterize the nature of the regulatory activities embedded in such regions and to obtain animal models to help dissect in vivo how variations in these regions contribute to human phenotypes.

Recent progress in whole genome chromatin profiling has led to the identification of chromatin features that are strongly correlated with gene regulatory elements [21-26], opening ways to obtain a comprehensive catalogue of these elements, and a better annotation of the regulatory genome [27]. Databases that document the in vivo activities of experimentally validated regulatory elements - mostly enhancers - further complement these approaches [28]. Such datasets on regulatory activity can be compared to gene expression data in

\section{Biomed Central}



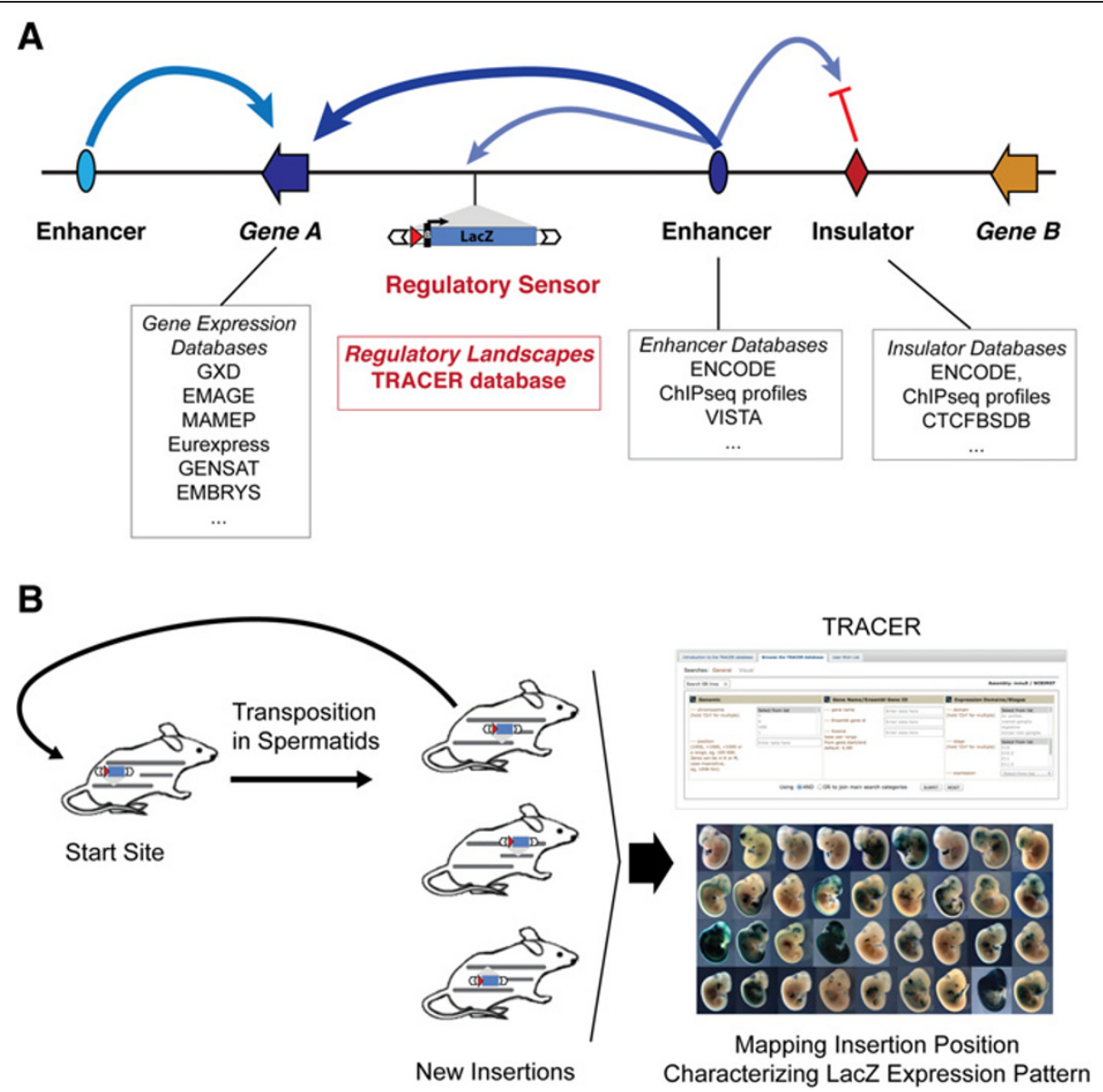

Figure 1 Genome organisation and TRACER. (A) Schematic representation of a genomic locus, with the different elements that control gene expression, and the specialized databases dedicated to their description (see Websites). The TRACER database displays information from a regulatory sensor that detects the regulatory influences active at its insertion site, outlining the characteristics and extent of genomic regulatory landscapes. (B) The GROMIT strategy. A transposon that contains the regulatory sensor can be remobilised in vivo from its start site by a transposase transgene (not shown) active only in the male germline [2]. The genomic position of the new insertions and the associated expression pattern defined by LacZ staining in mouse embryos is displayed in the TRACER database.

developing mouse embryos [29-35]. However one cannot reduce gene expression to a catalogue of the many potential regulatory elements present in the genome (from few hundred thousands to millions [22]). It is equally important to understand the interplay between the different elements present at a locus and how their different inputs are integrated and conveyed to target gene(s). Yet, compared to enhancers, other cis-regulatory elements such as silencers are much more elusive, despite their essential role in gene expression. Similarly important are the mechanisms that define the range and specificity of enhancer-promoter interactions. Indeed, changes in the relative position of genes and regulatory elements by chromosomal rearrangements and structural variations can alter gene expression with dramatic consequences [36-40]. Understanding these situations and the associated mechanisms requires approaches that complement the available catalogues of elements and provide a functional integrated view of the genome regulatory architecture.

For this purpose, we have developed an approach based on the distribution of a regulatory sensor gene throughout the mouse genome [2] (Figure 1B). The regulatory sensor consists of a LacZ reporter gene, which is driven by a minimal promoter that has no specific activity on its own but responds faithfully to endogenous enhancers. This regulatory sensor therefore uncovers the regulatory potential associated with a given genomic position, which results from the collective action of the different regulatory elements that act on this position. It thus reveals, in an operational manner, the gene regulatory activities within poorly characterized regions, or where annotation for activity is indirect (eg. chromatin profiling) or out of the proper genomic context (eg. transgenic assays). Importantly, the minimal promoter used does not display any obvious tissue- or 
enhancer-type bias, and the observed expression patterns often overlap with the ones of neighbouring genes [2]. The basic principle of the strategy is analogous to an enhancer-trap [41]; however, the sensor used in our approach has minimal impact on endogenous gene expression [2] and therefore reveals regulatory activities without titrating them away from their natural target genes.

This regulatory sensor is carried in a Sleeping Beauty transposon, which can be distributed randomly in the mouse genome, by remobilisation in the male germline [2]. Owing to the efficiency of this in vivo transposition system, we have recovered, identified and characterized a large number of insertions that provide a direct view of the regulatory activities associated with specific genomic regions. Furthermore, as the transposons used also carry a loxP site, the different lines can be used for in vivo chromosomal engineering, to generate mice with targeted deletions or duplications, or segmental aneuploidies [2,42-44]. The local hopping behaviour of Sleeping Beauty makes each line a potential starting point to scan a region of interest [45]: with our germline-specific transposase transgene, the remobilization rate ranges from 10 to $45 \%$, depending on the starting site, and more than $15 \%$ of new insertions are within $1 \mathrm{Mb}$ of the starting point. Thus, a research group with access to a limited number of cages can nonetheless set up a regional screen for its region of interest.

To provide a simple and useful access to the expression patterns and the mouse insertion strains generated with this on-going project, we have designed the Transposon- and Recombinase-Associated Chromosomal Engineering Resource (TRACER) database. This new database is freely accessible at http://tracerdatabase. embl.de/. It constitutes a substantial improvement over the previous one that was established to display the data from a limited pilot screen [2]. The new database comprises novel features that allow users to browse and perform refined searches of insertion sites by position and/ or expression patterns. The dataset is also now much larger (4-fold increase, with about 1500 insertions in July 2012), and is growing steadily. This web-based database not only provides information on regulatory activities present along the mouse genome but also gives access to a large collection of mice for engineering chromosomal rearrangements in non-genic intervals.

\section{Construction and content Dataset}

In July 2012, the TRACER database contained information on 1467 insertions, 643 of which had been characterized for expression in mouse embryos (mostly at stage E11.5). Specific expression patterns were reported for 344 insertions, documented and annotated by 852 pictures. The dataset is updated regularly, with new insertions and new expression data. Most insertions were obtained with SB9 or SB8 transposons, which contain the regulatory sensor and a $\operatorname{loxP}$ site cloned in one or the other orientation in the Sleeping Beauty transposon. Newer versions of the transposon with additional features have been developed (Figure 2) and will be introduced in the database when mice with such insertions will be available.

\section{Methodology and population of database}

All our data is stored on a MySQL 5.5.15 RDBMS community server (GPL). Server side programming languages are PHP and PERL CGI. CSS and the javascript framework jQuery render the client data display and graphical user interfaces.

As well as the external user interfaces described below, the TRACER database has internal interfaces restricted to contributing members and requiring login for authentication. These internal interfaces have all the LIMS (laboratory information management system) components

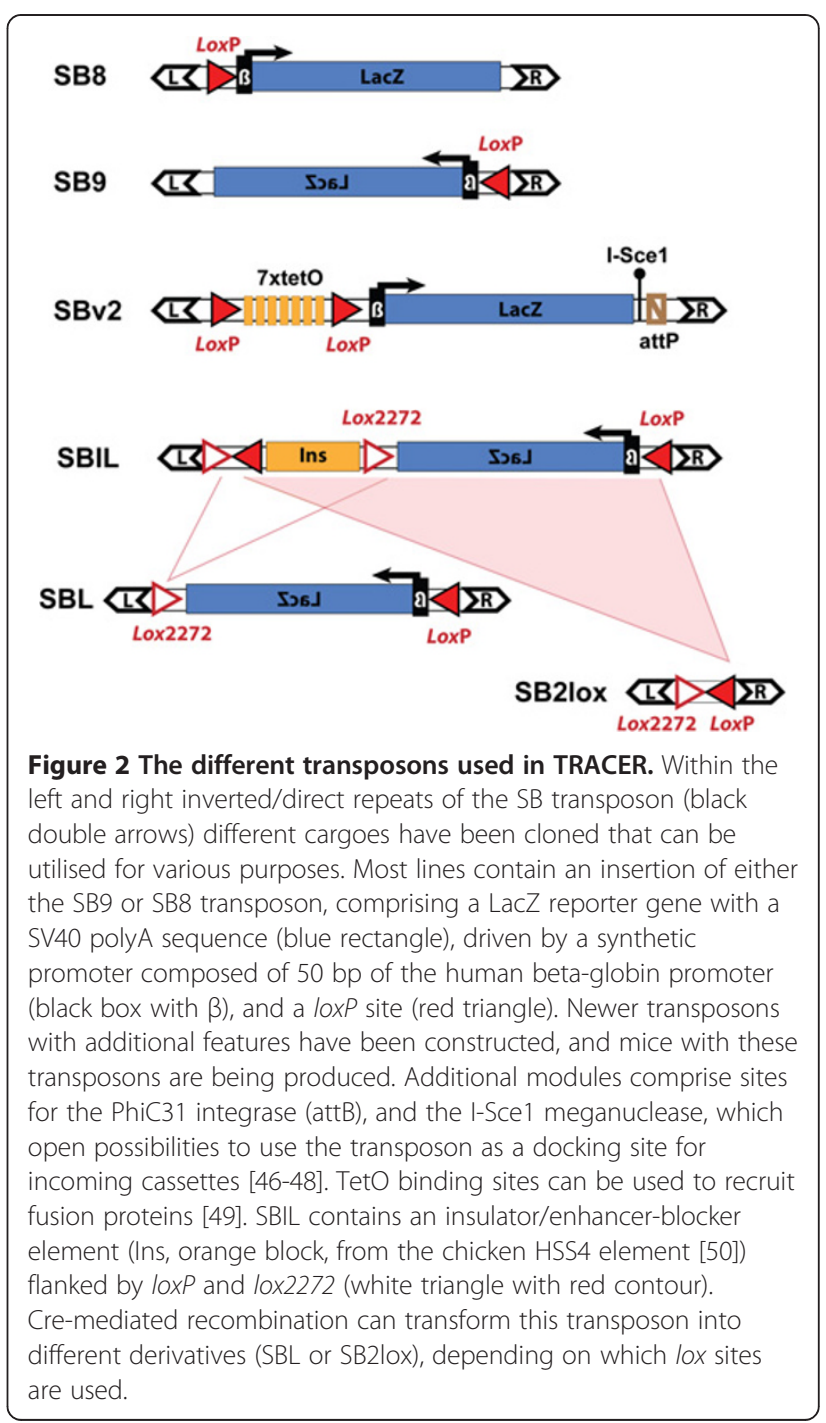


required for uploading data, curation of lines and various administration purposes.

The main internal interface allows lab staff to add all the text annotation, and insert sequence and image files associated with a particular TRACER line. There is also a batch upload interface for multiple insert sequences. The backend code automatically cuts the sequence down to just the insert, verifies the mutagen tag is present and the genomic sequence starts with 'TA'. The batch sequence submission tool is automatically coupled to the UCSC BLAT service with standard parameters (http:// genome.ucsc.edu/) to determine the best alignment and genomic location for each insert. When there are multiple good alignments, user intervention is possible to select the best genomic location. An input form is then populated for the aligned sequence along with any existing data for the line. A similar batch upload interface exists for the parsing of the expression image and annotation files. Internal users can also edit annotations for existing lines using a separate curation interface.

Many of the interfaces utilise a controlled vocabulary of terms to populate the drop-down menus, reducing the number of typos in the database and preserving the integrity of the data stored in TRACER. An administrative database exists to edit these controlled vocabularies.

The external interface allows users to register interest in particular lines, or - if the user's genomic region of interest is not yet covered - to wish for such a line when it becomes available. These requests are captured in the database and matching lines are displayed for the curators so they can contact the requesting researcher. For user-defined regions of interest, new matching lines are

\section{A}

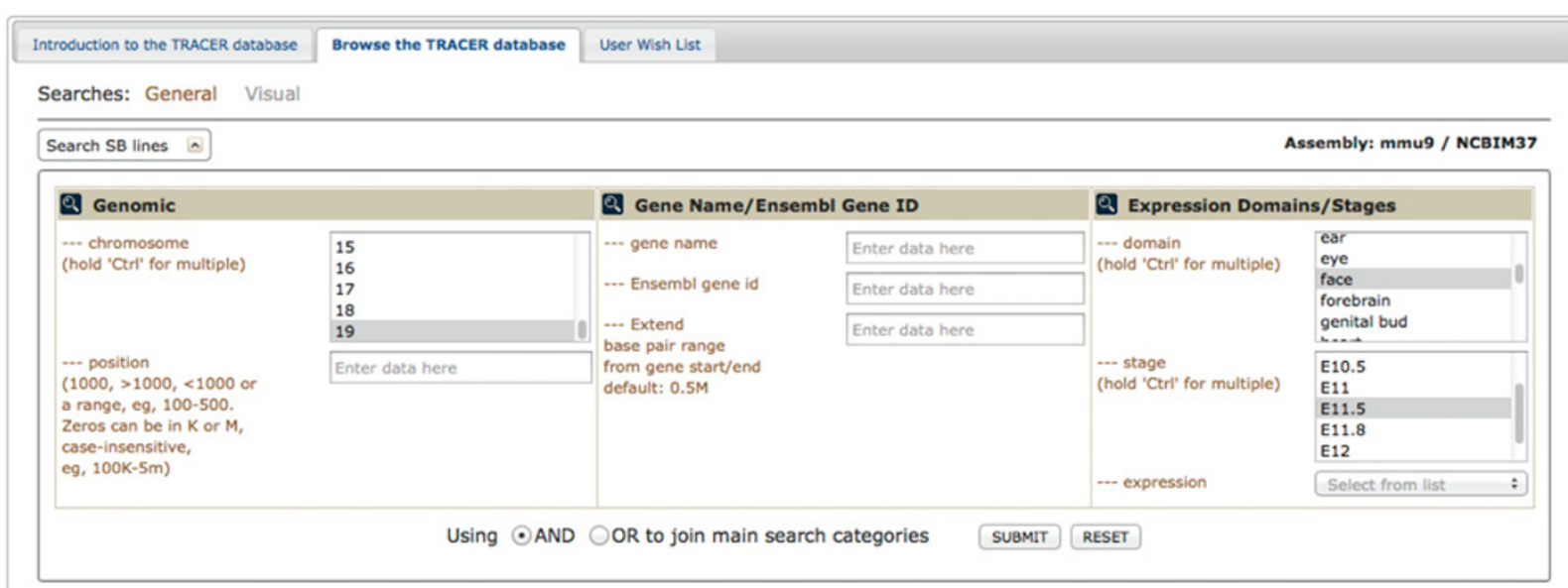

B

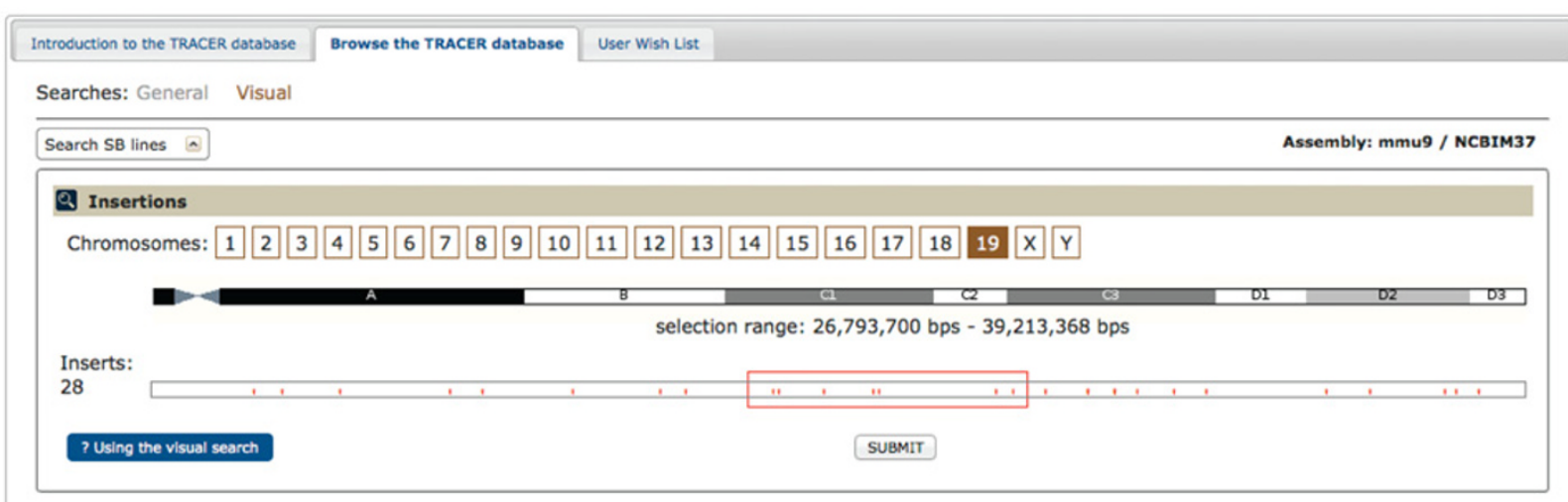

Figure 3 Searching the TRACER database. (A) TRACER lines can be retrieved ("General search") either based the genomic position of the insertion as defined by a genomic range or a region around a particular gene, or using the result of the expression assays, with the possibility to specify stage and expression domains of interest. (B) Alternatively ("Visual search"), the distribution of insertion sites along a chromosome is visualised, and those within the range defined by a dragable and expandable red rectangle will be returned. 


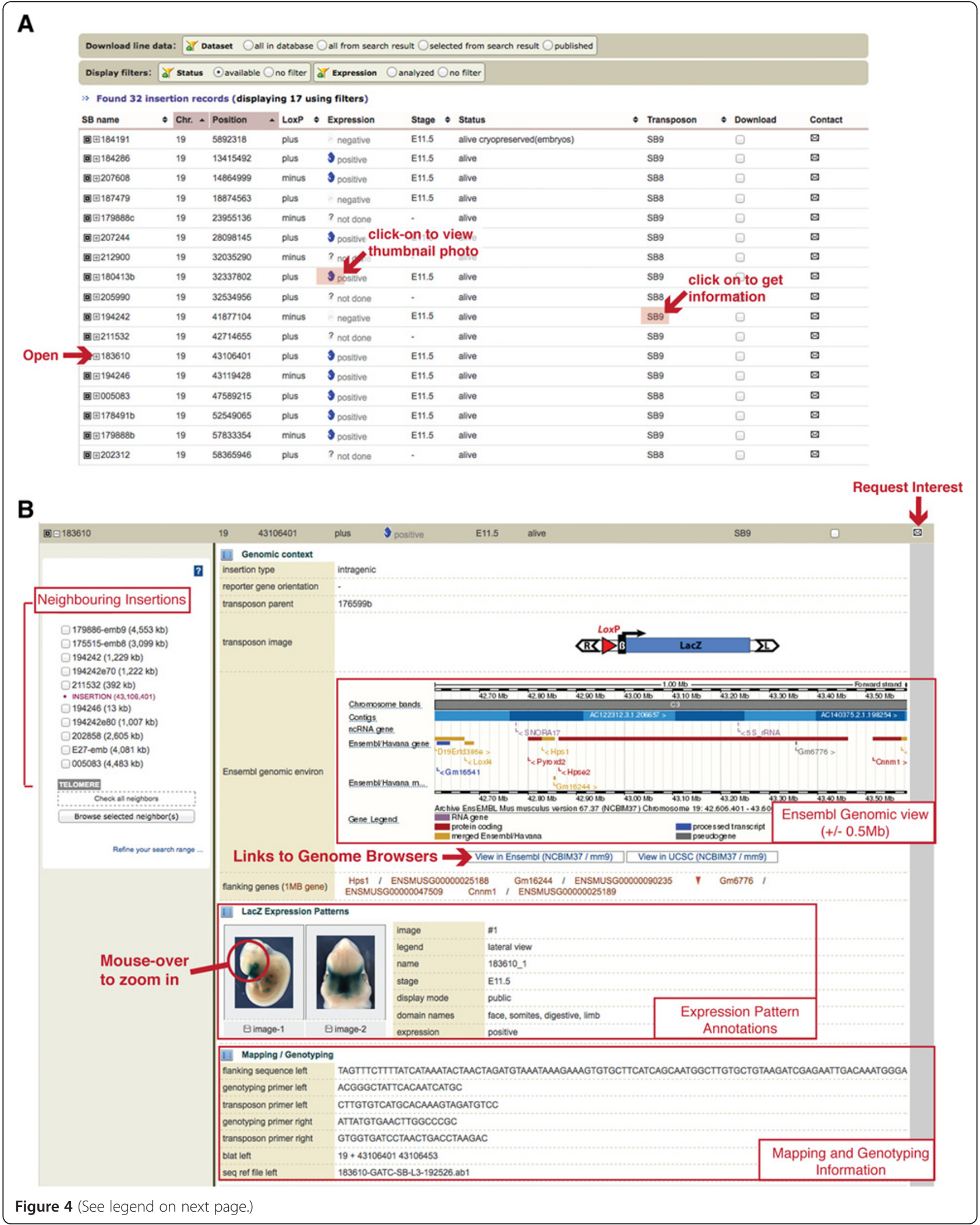


(See figure on previous page.)

Figure 4 Presentation of TRACER data. (A) Summary display of results for a TRACER search. For each line the TRACER name, genomic position, orientation of the loxP site, summary of expression (expressed/positive, not expressed/negative, not done; developmental stage(s) assayed), status (alive, cryopreserved, not maintained, newly created) and the transposon type are displayed. The final two columns allow users to select the data for download, or to register interest in the line. Clicking on the "open" icon gives access to the detailed information of an insertion of interest. Quick access to a thumbnail photo of a representative embryo and to the corresponding transposon is possible by a simple click on the corresponding zone (B) Detailed view of a TRACER mouse line. The top section reveals basic information about the insertion, including the type of insertion, the name of the parent insertion, the orientation of the loxP site and a visual representation of the transposon in the correct orientation. The first panel shows the genomic context of the insertion in a snapshot from the Ensembl genome browser (Ensembl Genomic view +/-0.5 Mb) along with links to view the insertion point in the Ensembl or UCSC genome browsers. The second panel shows photos and annotations of the expression patterns of the regulatory sensor. The images can be mouse-overed to reveal a high-resolution zoomed-in view, and the annotation of expression domains is displayed. The third panel shows information related to mapping and genotyping, including the sequence obtained from the mapping procedure, and the sequences of the primers used to genotype animals carrying this specific insertion. The interface in the panel on the left allows neighbouring insertions to be selected for detailed analysis of a region's regulatory potential.

automatically searched every week, or when triggered through the curator interface.

\section{Utility}

\section{Searching the TRACER database}

The "Browse the TRACER database" tab takes users to the main search interface of the website (Figure 3A). Insertions of interest can be identified by a variety of options. For genome-centric views, a genomic region of interest can be specified, defined either by chromosomal coordinates (reference genome is MGSCv37/mm9), or by a gene name ("associated gene name" from Ensembl database) and an optional user-defined flanking region (default is $0.5 \mathrm{Mb}$ ). In addition to this "General" option, one can perform a "Visual" search by clicking on the link at the top of the search window (Figure 3B). Users can view the distribution of insertions across each chromosome and drag a rectangle to define the region they want to retrieve lines. Searches can also be carried out based on expression patterns, using criteria such as positive/ negative, expression domains and expression stages. The label "negative" for expression means that no specific expression patterns was scored for this insertion at the embryonic stages assayed, whereas an insertion is labelled as "positive" if specific expression is detected at least at one stage of development. The majority of the insertions have been assayed at E11.5, but some data is available at other stages (E10 to E13). Expression domains are annotated using a simplified controlled vocabulary (e.g. branchial arches, cranial ganglia, digestive, dorsal root ganglia, ear, eye, face, forebrain, genital bud, heart, hindbrain, limb, midbrain, neural tube, somites, urogenital, others or widespread), which is compatible with the one used by the Vista Enhancer Database [28], in order to facilitate comparison of the two datasets.

\section{Display and download of data}

Results are returned as a table (Figure 4A) with one row per insertion and sortable columns displaying:
- The internal identifier of the mouse line in the TRACER database.

- The genomic position of the insertion (chr/position ; based on MGSCv37/mm9 genome assembly).

- The orientation of the loxP site in the transposon. "Plus" corresponds to the following orientation: centromere - 5'-ATAACTTCGTATA GCATACATTATACGAAGTTAT- 3 ' telomere. For comparison, loxP sites targeted by the International Knockout Mouse Consortium in genes transcribed from the plus strand (http://www.knockoutmouse. org/about/targeting-strategies) have the same orientation than TRACER "plus" loxP. Depending on the specific transposon, the orientation of the other features (transposon ends, reporter gene) varies: they are indicated and represented in the expanded view available by clicking on the "expand" icon.

- An icon and text, indicating whether expression analysis has been performed and whether LacZ reporter expression has been detected. The developmental stage(s) for which information is available are indicated in the next column. Expression assay is "positive" if the insertion showed LacZ staining at least at one of the stages assessed.

- The status of the insertion, indicating whether animals carrying the insertion are available. Insertions that were identified in F0 embryos, that couldn't be established from the founder or were discontinued, are labelled as "not maintained". Insertions "available" for further use or analysis fall under three categories: "alive" (line established with mice available in small numbers), "cryopreserved" (either as embryos or sperm) and "new" (usually corresponding to a new insertion, with only the founder animal). The status of an insertion is dynamic: not all "new" insertions are established, and depending on circumstances, "alive" ones may become "cryopreserved" or "not maintained".

- Transposon type: most of the available lines harbour a simple regulatory sensor with a lacZ reporter and 
a single $\operatorname{lox} P$ site, in one or the other orientation relative to the transposon ends (SB8 and SB9). New transposons with additional features have been constructed (see Figure 2), and lines containing them are being established and will be added to the resource. Detailed maps and sequences of available transposons are available on the Tracer website.

The final two columns display a checkbox to download the complete set of information available for an insertion, and an email link to indicate interest in a specific insertion. The toolbar buttons above the results table can be used to filter the search results, and to show only available lines and/or lines with expression data.

Further details on a given insertion can be seen by clicking the expand icon next to each record (Figure 4B). The first section describes the genomic context of the insertion. It lists whether the insertion is located in a gene desert (a gene-free region larger than $500 \mathrm{~kb}$ ), intergenic (less than $500 \mathrm{~kb}$-long), intronic or exonic region, specifies the orientation of the reporter gene, and the parental insertion line from which the insertion was obtained. This section also contains a schematic of the transposon construct, the genomic environment and flanking genes in a snapshot from the Ensembl genome browser [51] along with links to view the insertion point in Ensembl or the UCSC genome browser [52].

The second section shows the LacZ expression patterns obtained for the insertion, when available. Mousing over each thumbnail image show a zoomed-in, trackable high-resolution view of the image. In addition, the stage and viewpoint of the image is recorded along with annotations using the expression domain categories detailed above. One can switch from one image to another one by clicking on the corresponding thumbnail.

The final section shows details regarding how the genomic position of the insertion was determined, such as the flanking sequence(s) obtained (trimmed to the TA dinucleotide duplicated upon Sleeping Beauty insertion [53]), and where this sequence mapped where this sequence mapped to genome using BLAT [54]. When available, primers that have been used to genotype embryos and mice for this specific insertion are indicated.

The left hand panel of the expanded section contains an interface that displays lines with insertion points within $5 \mathrm{Mb}$ (or a user-selected range) (Figure 4B). Users can select one or more of these lines, and open a new tab displaying these flanking lines. This feature is particularly useful to compare regulatory activities across large regions, and to delineate the extent of regulatory domains.

Finally, the toolbar below the search interface allows data to be downloaded for the whole TRACER database, the search results, user selected lines or just the lines described in publications referring to the dataset. Additionally, all available images can be downloaded. Requests for higher resolution photos and other questions can be sent to gromit@embl.de. Most LacZ stained embryos has been archived, albeit in limited numbers for each insertion, and may be made available upon request.

\section{User wish list}

Although the TRACER database already covers a substantial proportion of the genome, it is likely that individual researchers will be interested to get information and mice with transposons in regions where we haven't yet identified an insertion. Given the high efficiency of transposition, the number of new insertions identified in on-going remobilisation efforts ( $\sim 10$ per week) exceeds our current capacity to keep, expand and cryopreserve all of them (Figure 5). The "User wish list" tab allows scientists to indicate particular genomic regions they are interested in, along with their contact details (Figure 5C). Once an insertion in this region is identified, it is "flagged" for the producing group, so that the corresponding animal is kept, and the interested group will be contacted.

\section{Discussion}

\section{A functional view of the genome with TRACER}

The introduction of a "regulatory sensor" in the genome provides a direct operational readout of the activities that can contribute to gene expression, which surround the insertion point. Similar enhancer-trap screens have widely been used in Drosophila [41] and to some extent in zebrafish [55-58], providing information about genes and genomes, as well as a series of useful markers and tools. Their use in mice has been limited $[59,60]$, in part due to the low throughput of transgenesis, and technical difficulties of generating single-copy insertions. The development of robust and efficient in vivo transposition systems [2,61-63], as shown here, or the use of lentiviral transgenesis, as recently described elsewhere [64], open new exciting possibilities to conduct such screens in an efficient and affordable manner.

Collections of insertions generated by these approaches can provide useful information and tools, and the TRACER database represents a substantial step to capitalise on such a collection, by centralizing and giving access to data and to mouse lines. We present and discuss here briefly some of the possible uses of this database and of the information therein (Figure 6).

By querying the database for a gene or a region of interest, one can identify expression patterns and regulatory activities associated with that location and its surroundings. The observed activity may indicate possible developmental or tissue-specific regulation of genes, and shed light on their physiological roles in vivo (Figure 6A). 


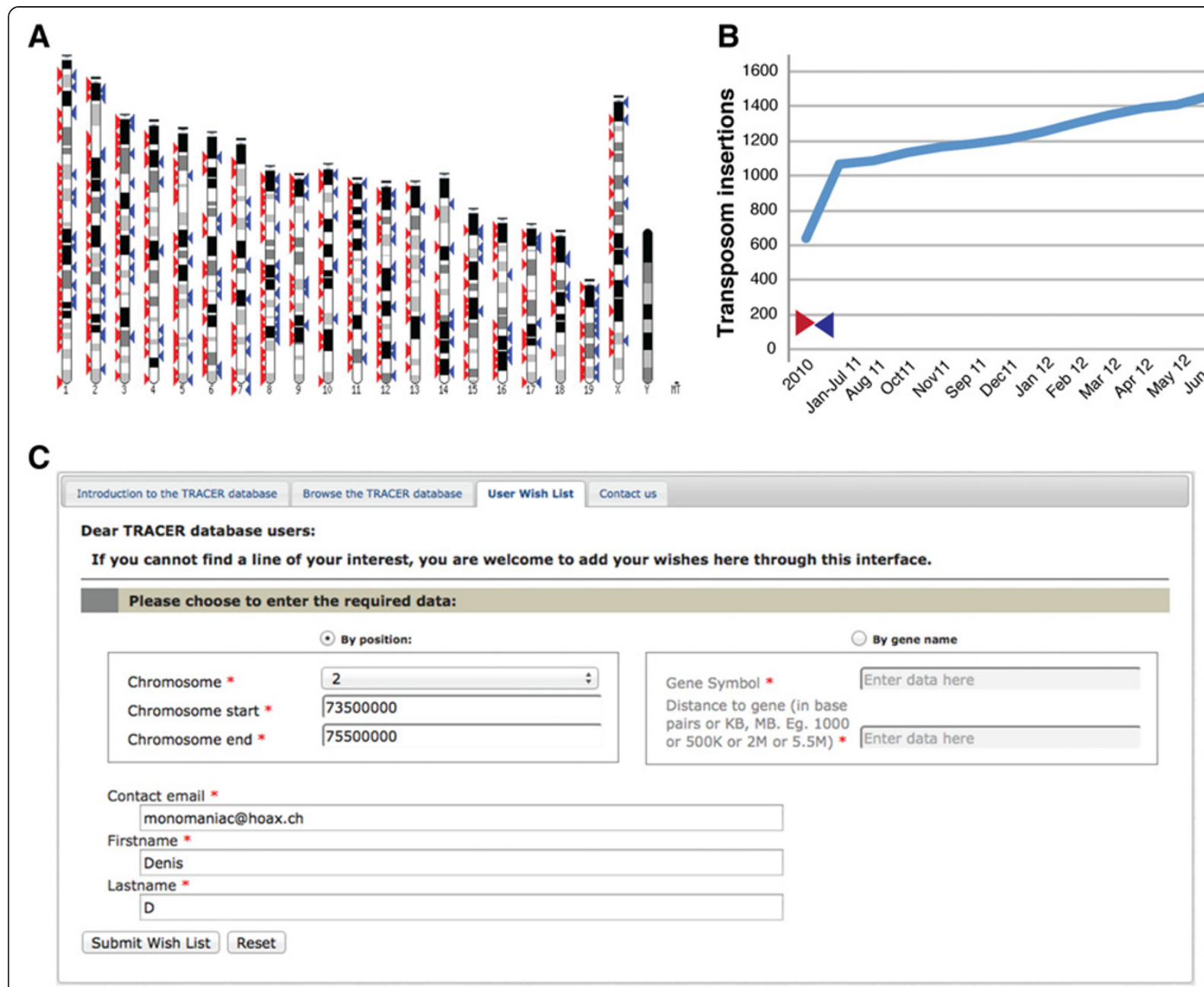

Figure 5 Evolution of TRACER dataset. (A) Genomic distribution of the insertions with expression annotations (red) and available as lines (blue) (generated with the Karyoview tool from Ensembl - status in July 2012). Insertions on the Y chromosome are not shown as their positions are ambiguous (B) The number of mapped insertions increases at a regular pace (light blue line), almost paralleled by expression reports (red cursor). A smaller proportion of these are kept alive or archived by cryopreservation (blue cursor). (C) The user wish list allows researchers to register interest for a genomic interval. It ensures that mice with new insertions in this region will be preserved for the scientist who indicated this request. The interval of interest can be defined by position or by gene name.

However, we wish to emphasize that the regulatory sensor sometimes reflects only a subset of the expression domains of a given gene [2]. Although the sensor responds accurately to influences from long-range remote enhancers, it is less likely to capture the input of promoter elements that have a limited range of action: tissue-restricted expression of the sensor may therefore represent a tissue-specific modulation of an otherwise broadly expressed gene; yet, this modulation may correspond to important biological functions.

Also, the expression pattern associated with an insertion does not necessarily imply that a corresponding enhancer lies nearby, as illustrated by the shared expression of distant insertions (Figure 6A,C; other examples in [2]). Instead, the sensor reports the collective input at a given position of both positive and negative regulatory elements. Accordingly, comparing the expression pattern of neighbouring insertions to each other and to known enhancer activities $[21,65]$ can reveal important regulatory features. These include the range of action of enhancers, the boundaries of expression domains, the presence of silencers or other repressive or insulating elements that modulate enhancer activity and cannot be obtained from other types of datasets and approaches. In essence, TRACER provides an operational view of the regulatory structure of the mammalian genome, and delineates the extent of the large regulatory landscapes [6] that subdivide the genome into functional units. It constitutes a functional counterpart to views obtained by different methods; including, for example, Genome Regulatory Blocks that are delineated by the density of conserved non-coding elements and synteny conservation 


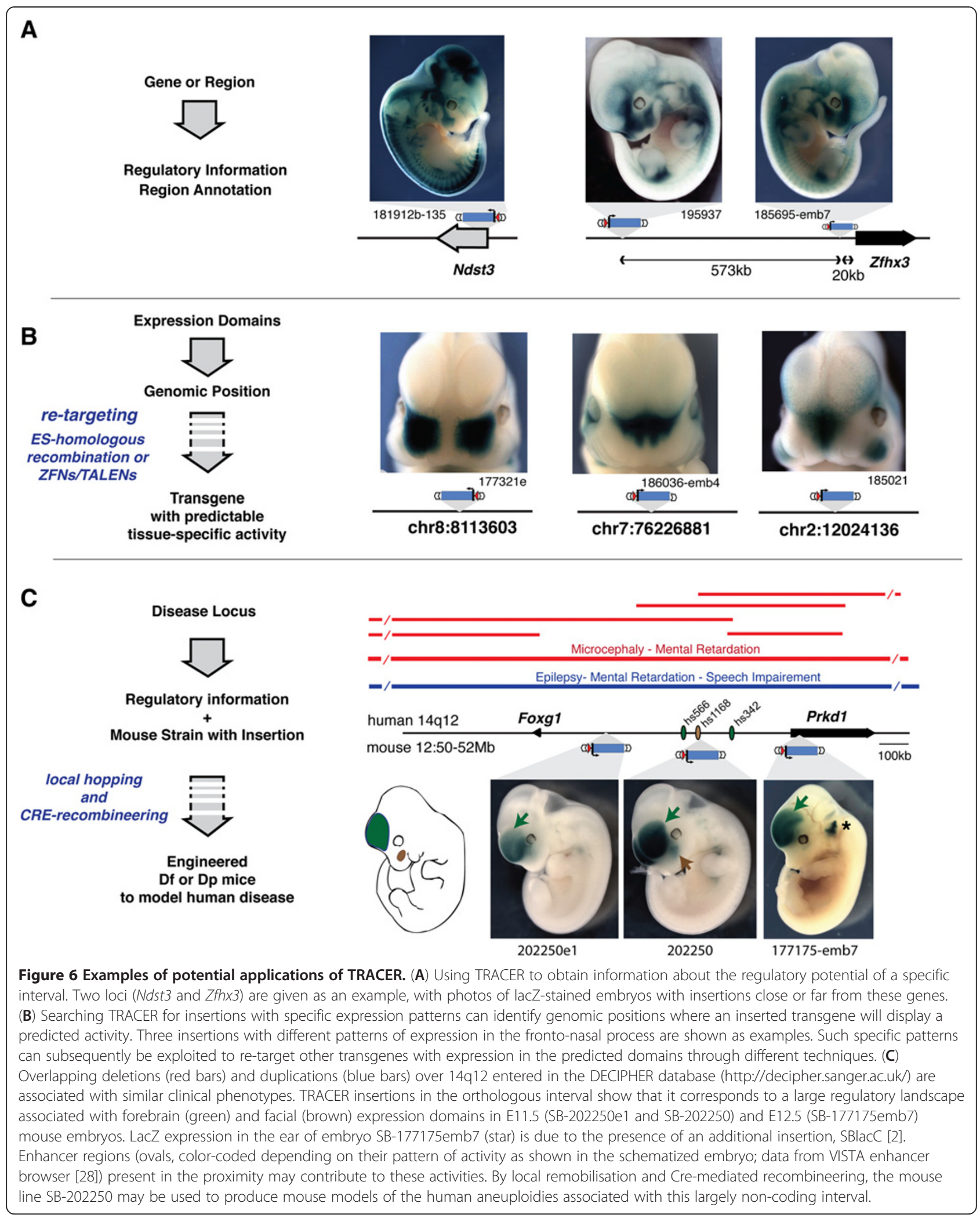


[66,67], Topological Associated Domains defined by chromosomal interaction biases [68,69], and EnhancerPromoter Units that are revealed by clusters of coincident promoter-enhancer chromatin signatures [70].

The data present in TRACER identifies genomic positions where an inserted transgene will adopt a highly specific expression profile (Figure 6B). Transgenes that drive the expression of markers to label specific cells (such as fluorescent markers) or of effector genes (for example Cre recombinase) in defined cell-types or embryonic tissues have proven very useful to dissect biological and genetic processes. "Position-effects" (the action of endogenous regulatory elements on transgenes) are usually considered as a problem for transgenic experiments because they lead to partially unpredictable outcomes. With the information displayed in TRACER, one can instead exploit position effects, and select genomic sites that will convey an expression pattern of interest. Importantly, many of these sites are located far from genes, implying that their use would have less functional impact than a gene knock-in. The sensor integrates the inputs of both enhancers and silencers that are acting at its position: consequently, the observed pattern is often more restricted than the one driven by enhancer-only constructs or displayed by the neighbouring genes [2]. Hence, retargeting positions identified in TRACER with a transgene of interest should provide a reliable method to create new tissue- and cell-type specific transgenes. This can be done by homologous recombination in mouse ES cells, but the rapid development of Zinc-Finger or TALE Nuclease-associated targeted transgenesis may offer more efficient alternatives [46,71,72].

In addition to maps of genomic "regulatory landscapes", TRACER provides access to a large and growing collection of mice with different transposon insertions (around 200 in July 2012). Only few insertions are likely to disrupt genes or key/highly conserved regulatory elements directly. Instead, these mice can be used for other purposes, and in particular for engineering aneuploidies and structural variants. Chromosomal aneuploidies are often found in patients suffering developmental malformations and/or neuropsychiatric disorders. In some cases, single gene-knockout can reproduce the phenotypes observed in human patients; however, for numerous other conditions, such as contiguous gene diseases, chromosomal duplications or rearrangements in noncoding intervals, gene-based alleles do not provide accurate models. Because Sleeping Beauty transposons frequently re-insert in the vicinity of their initial position, it is possible to use one insertion in a region of interest to generate additional local re-insertions. These insertions can be (re)combined owing to the associated loxP sites, to produce a series of rearrangements of this locus that model genomic alterations found in human patients, and help determine the causal elements or genes (Figure 6C). Such a use of the TRACER resource and GROMIT strategy can be particularly well suited for large gene clusters (eg. proto-cadherins, KRAB-zinc finger genes, olfactory receptors) or gene-deserts associated with human pathologies, complementing the genecentric resource provided by the International Knockout Mouse Consortium. Given the growing recognition of the biological importance of genomic structural variants for human diseases, we anticipate that TRACER will be a useful resource to rapidly engineer allelic series of structural variants in mouse orthologous intervals, helping to create novel models of human genomic disorders.

\section{Conclusion}

\section{TRACER database and community}

Owing to the dynamic nature of transposon elements, the resource present in TRACER will expand steadily with the number of users. Each lab using this transposon technology to investigate a region of interest by "local" hopping will produce a substantial number of by-products ( 80\% of the new insertions). Even if these insertions may not be useful for the producing lab, they can be of interest for others. TRACER is designed to serve as a central "virtual" repository to share those mice. Further information, including references, detailed maps and sequences of the different transposons and transgenes in use, and protocols for mapping of new insertions are available through the pages of the TRACER website.

To facilitate exchanges, the TRACER database incorporates several features and internal interfaces for contributing groups (automated insertion mapping, annotation and administration). In particular, the "User wish list" feature offers a simple manner to readily "tag" newly generated mice of interest without a major investment or commitment of the producing labs.

\section{Availability and requirements}

The database is accessible at the web addresses:

http://tracerdatabase.embl.de

http://www.ebi.ac.uk/panda-srv/tracer/index.php

Websites - links

ENSEMBL: http://www.ensembl.org/Mus_musculus/Info/ Index

UCSC Genome Browser: http://genome.ucsc.edu/index. html

CTCFBSBD: http://insulatordb.uthsc.edu/

VISTA: http://enhancer.lbl.gov/frnt_page_n.shtml

GXD: http://www.informatics.jax.org/mgihome/GXD/ aboutGXD.shtml

EMAGE: http://www.emouseatlas.org/emage/

GENSAT: http://www.gensat.org/index.html

MAMEP: http://mamep.molgen.mpg.de/index.php 


\section{EUREXPRESS: http://www.eurexpress.org/ EMBRYS: http://embrys.jp/embrys/html/MainMenu.html DECIPHER: http://decipher.sanger.ac.uk/}

\section{Competing interests}

The authors declare that they have no competing interest.

\section{Authors' contributions}

CKC, DS and FS designed the database with critical input from OS, WU, TT, SR. CKC wrote the code, the different interfaces and tools associated with TRACER. OS, WU, TT, SR and FS provided all data present in the database. DS and FS wrote the manuscript, with input and suggestions from all the other authors. All authors read and approved the final manuscript.

\section{Acknowledgements}

The authors thank the members of the EMBL Laboratory Animal Resource, in particular Andrea Schulz, Silke Feller, Michaela Wesch and Klaus Schmitt; the development and maintenance of this mouse resource would not be possible without their dedication and constant support. We thank as well Manuela Borchert and Anne Hermelin for advice and design of the TRACER webpages. The TRACER database and resource is funded by EMBL. The production of some of the strains described in the database was made in the course of projects supported by the European Commission-FP7 (grant Health 223210/CISSTEM) and Human Frontier Science Program (grant RGY0081/2008-C) to FS.

\section{Author details}

${ }^{1}$ European Bioinformatics Institute - European Molecular Biology Laboratory, Wellcome Trust Genome Campus, Hinxton, Cambridge CB10 1SD, UK. ${ }^{2}$ Developmental Biology Unit - European Molecular Biology Laboratory, Heidelberg, Germany. ${ }^{3}$ Present address: Welcome Trust Sanger Institute, Wellcome Trust Genome Campus, Hinxton, Cambridge CB10 1SD, UK.

Received: 31 October 2012 Accepted: 22 March 2013

Published: 2 April 2013

\section{References}

1. Alexander RP, Fang G, Rozowsky J, Snyder M, Gerstein MB: Annotating noncoding regions of the genome. Nat Rev Genet 2010, 11:559-571.

2. Ruf S, Symmons O, Uslu W, Dolle D, Hot C, Ettwiller L, Spitz F: Large-scale analysis of the regulatory architecture of the mouse genome with a transposon-associated sensor. Nat Genet 2011, 43:379-386.

3. Visel A, Rubin EM, Rubin EM, Pennacchio LA, Pennacchio LA: Genomic views of distant-acting enhancers. Nature 2009, 461:199-205.

4. Jeong Y, El-Jaick K, Roessler E, Muenke M, Epstein DJ: A functional screen for sonic hedgehog regulatory elements across a $1 \mathrm{Mb}$ interval identifies long-range ventral forebrain enhancers. Development 2006, 133:761-772.

5. Zuniga A, Michos O, Spitz F, Haramis A-PG, Panman L, Galli A, Vintersten K, Klasen C, Mansfield W, Kuc S, Duboule D, Dono R, Zeller R: Mouse limb deformity mutations disrupt a global control region within the large regulatory landscape required for Gremlin expression. Genes Dev 2004, 18:1553-1564.

6. Spitz F, Gonzalez F, Duboule D: A global control region defines a chromosomal regulatory landscape containing the HoxD cluster. Cell 2003, 113:405-417.

7. Montavon T, Soshnikova N, Mascrez B, Joye E, Thevenet L, Splinter E, de Laat W, Spitz F, Duboule D: A regulatory archipelago controls Hox genes transcription in digits. Cell 2011, 147:1132-1145.

8. Nobrega MA, Ovcharenko I, Afzal V, Rubin EM: Scanning human gene deserts for long-range enhancers. Science 2003, 302:413.

9. Lettice LA, Heaney SJH, Purdie LA, Li L, de Beer P, Oostra BA, Goode D, Elgar $G$, Hill RE, de Graaff E: A long-range Shh enhancer regulates expression in the developing limb and fin and is associated with preaxial polydactyly. J Neurosci Res 2003, 12:1725-1735.

10. Uchikawa M, Ishida Y, Takemoto T, Kamachi Y, Kondoh H: Functional analysis of chicken Sox2 enhancers highlights an array of diverse regulatory elements that are conserved in mammals. Dev Cell 2003, 4:509-519.

11. Kleinjan DA, Seawright A, Mella S, Carr CB, Tyas DA, Simpson TI, Mason JO, Price DJ, van Heyningen V: Long-range downstream enhancers are essential for Pax6 expression. Dev Biol 2006, 299:563-581.
12. Lettice LA, Horikoshi T, Heaney SJH, van Baren MJ, van der Linde HC, Breedveld GJ, Joosse M, Akarsu N, Oostra BA, Endo N, Shibata M, Suzuki M, Takahashi E, Shinka T, Nakahori Y, Ayusawa D, Nakabayashi K, Scherer SW, Heutink P, Hill RE, Noji S: Disruption of a long-range cis-acting regulator for Shh causes preaxial polydactyly. Proc Natl Acad Sci USA 2002, 99:7548-7553.

13. Kleinjan DA, van Heyningen V: Long-range control of gene expression: emerging mechanisms and disruption in disease. Am J Hum Genet 2005, 76:8-32.

14. Jeong Y, Leskow FC, El-Jaick K, Roessler E, Muenke M, Yocum A, Dubourg C, Li X, Geng X, Oliver G, Epstein DJ: Regulation of a remote Shh forebrain enhancer by the Six3 homeoprotein. Nat Genet 2008, 40:1348-1353.

15. Benko S, Fantes JA, Amiel J, Kleinjan D-J, Thomas S, Ramsay J, Jamshidi N, Essafi A, Heaney S, Gordon CT, McBride D, Golzio C, Fisher M, Perry P, Abadie V, Ayuso C, Holder-Espinasse M, Kilpatrick N, Lees MM, Picard A, Temple IK, Thomas P, Vazquez M-P, Vekemans M, Crollius HR, Hastie ND, Munnich A, Etchevers HC, Pelet A, Farlie PG, et al: Highly conserved noncoding elements on either side of SOX9 associated with Pierre Robin sequence. Nat Genet 2009, 41:359-364.

16. Rahimov F, Marazita ML, Visel A, Cooper ME, Hitchler MJ, Rubini M, Domann FE, Govil M, Christensen K, Bille C, Melbye M, Jugessur A, Lie RT, Wilcox AJ, Fitzpatrick DR, Green ED, Mossey PA, Little J, Steegers-Theunissen RP, Pennacchio LA, Schutte BC, Murray JC: Disruption of an AP-2alpha binding site in an IRF6 enhancer is associated with cleft lip. Nat Genet 2008, 40:1341-1347.

17. Tuupanen S, Turunen M, Lehtonen R, Hallikas O, Vanharanta S, Kivioja T, Björklund M, Wei G, Yan J, Niittymäki I, Mecklin J-P, Järvinen H, Ristimäki A, Di-Bernardo M, East P, Carvajal-Carmona L, Houlston RS, Tomlinson I, Palin K, Ukkonen E, Karhu A, Taipale J, Aaltonen LA: The common colorectal cancer predisposition SNP rs6983267 at chromosome 8q24 confers potential to enhanced Wnt signaling. Nat Genet 2009, 41:885-890.

18. Wasserman NF, Aneas I, Nobrega MA: An 8q24 gene desert variant associated with prostate cancer risk confers differential in vivo activity to a MYC enhancer. Genome Res 2010, 20:1191-1197.

19. Visser M, Kayser M, Palstra RJ: HERC2 rs12913832 modulates human pigmentation by attenuating chromatin-loop formation between a longrange enhancer and the OCA2 promoter. Genome Res 2012, 22:446-455.

20. Harismendy O, Notani D, Song X, Rahim NG, Tanasa B, Heintzman N, Ren B, Fu X-D, Topol EJ, Rosenfeld MG, Frazer KA: 9p21 DNA variants associated with coronary artery disease impair interferon- $\gamma$ signalling response. Nature 2011, 470:264-268.

21. Visel A, Blow MJ, Li Z, Zhang T, Akiyama JA, Holt A, Plajzer-Frick I, Shoukry M, Wright C, Chen F, Afzal V, Ren B, Rubin EM, Pennacchio LA: ChIP-seq accurately predicts tissue-specific activity of enhancers. Nature 2009, 457:854-858.

22. Heintzman ND, Hon GC, Hawkins RD, Kheradpour P, Stark A, Harp LF, Ye Z, Lee LK, Stuart RK, Ching CW, Ching KA, Antosiewicz-Bourget JE, Liu H, Zhang X, Green RD, Lobanenkov W, Stewart R, Thomson JA, Crawford GE, Kellis $M$, Ren B: Histone modifications at human enhancers reflect global cell-type-specific gene expression. Nature 2009, 459:108-112.

23. Creyghton MP, Cheng AW, Welstead GG, Kooistra T, Carey BW, Steine EJ, Hanna J, Lodato MA, Frampton GM, Sharp PA, Boyer LA, Young RA, Jaenisch R: Histone H3K27ac separates active from poised enhancers and predicts developmental state. Proc Natl Acad Sci USA 2010, 107:21931-21936.

24. Rada-Iglesias A, Bajpai R, Swigut T, Brugmann SA, Flynn RA, Wysocka J: A unique chromatin signature uncovers early developmental enhancers in humans. Nature 2011, 470:279-283.

25. Li G, Ruan X, Auerbach RK, Sandhu KS, Zheng M, Wang P, Poh HM, Goh Y, Lim J, Zhang J, Sim HS, Peh SQ, Mulawadi FH, Ong CT, Orlov YL, Hong S, Zhang Z, Landt S, Raha D, Euskirchen G, Wei C-L, Ge W, Wang H, Davis C, Fisher-Aylor Kl, Mortazavi A, Gerstein M, Gingeras T, Wold B, Sun Y, et al: Extensive promoter-centered chromatin interactions provide a topological basis for transcription regulation. Cell 2012, 148:84-98.

26. Ernst J, Kheradpour P, Mikkelsen TS, Shoresh N, Ward LD, Epstein CB, Zhang X, Wang L, Issner R, Coyne M, Ku M, Durham T, Kellis M, Bernstein BE: Mapping and analysis of chromatin state dynamics in nine human cell types. Nature 2011, 473:43-49.

27. Consortium TEP: An integrated encyclopedia of DNA elements in the human genome. Nature 2012, 489:57-74.

28. Visel A, Minovitsky S, Dubchak I, Pennacchio LA: VISTA Enhancer Browser-a database of tissue-specific human enhancers. Nucleic Acids Res 2007, 35:D88-92. 
29. Finger $\mathrm{JH}$, Smith CM, Hayamizu TF, McCright IJ, Eppig JT, Kadin JA, Richardson JE, Ringwald M: The mouse Gene Expression Database (GXD): 2011 update. Nucleic Acids Res 2011, 39:D835-41.

30. Heintz N: Gene expression nervous system atlas (GENSAT). Nat Neurosci 2004, 7:483.

31. Yokoyama S, Ito Y, Ueno-Kudoh H, Shimizu H, Uchibe K, Albini S, Mitsuoka K, Miyaki S, Kiso M, Nagai A, Hikata T, Osada T, Fukuda N, Yamashita S, Harada D, Mezzano V, Kasai M, Puri PL, Hayashizaki Y, Okado H, Hashimoto $\mathrm{M}$, Asahara $\mathrm{H}$ : A systems approach reveals that the myogenesis genome network is regulated by the transcriptional repressor RP58. Dev Cell 2009, 17:836-848

32. Richardson L, Venkataraman S, Stevenson P, Yang Y, Burton N, Rao J, Fisher M, Baldock RA, Davidson DR, Christiansen JH: EMAGE mouse embryo spatial gene expression database: 2010 update. Nucleic Acids Res 2010, 38: D703-9.

33. Visel A, Thaller C, Eichele G: GenePaint.org: an atlas of gene expression patterns in the mouse embryo. Nucleic Acids Res 2004, 32:D552-6.

34. Diez-Roux G, Banfi S, Sultan M, Geffers L, Anand S, Rozado D, Magen A, Canidio E, Pagani M, Peluso I, Lin-Marq N, Koch M, Bilio M, Cantiello I, Verde R, De Masi C, Bianchi SA, Cicchini J, Perroud E, Mehmeti S, Dagand E, Schrinner S, Nürnberger A, Schmidt K, Metz K, Zwingmann C, Brieske N, Springer C, Hernandez AM, Herzog S, et al: A high-resolution anatomical atlas of the transcriptome in the mouse embryo. PLoS Biol 2011, 9:e1000582.

35. Neidhardt L, Gasca S, Wertz K, Obermayr F, Worpenberg S, Lehrach H, Herrmann BG: Large-scale screen for genes controlling mammalian embryogenesis, using high-throughput gene expression analysis in mouse embryos. Mech Dev 2000, 98:77-94.

36. Klopocki E, Ott C, Benatar N, Ullmann R, Mundlos S, Lehmann K: A microduplication of the long range $\mathrm{SHH}$ limb regulator (ZRS) is associated with triphalangeal thumb-polysyndactyly syndrome. J Med Genet 2008, 45:370-375.

37. Sun M, Ma F, Zeng X, Liu Q, Zhao X, Wu F, Wu G, Zhang Z, Gu B, Zhao Y, Tian S, Lin B, Kong X, Zhang X, Yang W, Lo W: Triphalangeal thumbpolysyndactyly syndrome and syndactyly type IV are caused by genomic duplications involving the long-range, limb-specific $\mathrm{SHH}$ enhancer. J Med Genet 2008, 45:589-595.

38. Dathe K, Kjaer KW, Brehm A, Meinecke P, Nürnberg P, Neto JC, Brunoni D, Tommerup N, Ott CE, Klopocki E, Seemann P, Mundlos S: Duplications involving a conserved regulatory element downstream of BMP2 are associated with brachydactyly type A2. Am J Hum Genet 2009, 84:483-492

39. Dimitrov Bl, de Ravel T, Van Driessche J, de Die-Smulders C, Toutain A, Vermeesch JR, Fryns JP, Devriendt K, Debeer P: Distal limb deficiencies, micrognathia syndrome, and syndromic forms of split hand foot malformation (SHFM) are caused by chromosome $10 \mathrm{q}$ genomic rearrangements. J Med Genet 2010, 47:103-111.

40. Kurth I, Klopocki E, Stricker S, van Oosterwijk J, Vanek S, Altmann J, Santos HG, van Harssel JJT, de Ravel T, Wilkie AOM, Gal A, Mundlos S: Duplications of noncoding elements 5 ' of SOX9 are associated with brachydactylyanonychia. Nat Genet 2009, 41:862-863.

41. Bellen HJ: Ten years of enhancer detection: lessons from the fly. Plant Cell 1999, 11:2271-2281.

42. Hérault $Y$, Rassoulzadegan M, Cuzin F, Duboule D: Engineering chromosomes in mice through targeted meiotic recombination (TAMERE). Nat Genet 1998, 20:381-384.

43. Wu S, Ying G, Wu Q, Capecchi MR: Toward simpler and faster genomewide mutagenesis in mice. Nat Genet 2007, 39:922-930.

44. Spitz F, Herkenne C, Morris MA, Duboule D: Inversion-induced disruption of the Hoxd cluster leads to the partition of regulatory landscapes. Nat Genet 2005, 37:889-893.

45. Keng WW, Yae K, Hayakawa T, Mizuno S, Uno Y, Yusa K, Kokubu C, Kinoshita T, Akagi K, Jenkins NA, Copeland NG, Horie K, Takeda J: Region-specific saturation germline mutagenesis in mice using the Sleeping Beauty transposon system. Nat Methods 2005, 2:763-769.

46. Meyer M, de Angelis MH, Wurst W, Kühn R: Gene targeting by homologous recombination in mouse zygotes mediated by zinc-finger nucleases. Proc Natl Acad Sci USA 2010, 107:15022-15026.

47. Venken KJT, Schulze KL, Haelterman NA, Pan H, He Y, Evans-Holm M, Carlson JW, Levis RW, Spradling AC, Hoskins RA, Bellen HJ: MiMIC: a highly versatile transposon insertion resource for engineering Drosophila melanogaster genes. Nat Methods 2011, 8:737-743.
48. Smih F, Rouet P, Romanienko PJ, Jasin M: Double-strand breaks at the target locus stimulate gene targeting in embryonic stem cells. Nucleic Acids Res 1995, 23:5012-5019.

49. Groner AC, Meylan S, Ciuffi A, Zangger N, Ambrosini G, Dénervaud N, Bucher $\mathrm{P}$, Trono D: KRAB-zinc finger proteins and KAP1 can mediate long-range transcriptional repression through heterochromatin spreading. PLoS Genet 2010, 6:e1000869.

50. Chung $\mathrm{JH}$, Whiteley M, Felsenfeld G: A 5 ' element of the chicken beta-globin domain serves as an insulator in human erythroid cells and protects against position effect in Drosophila. Cell 1993, 74:505-514.

51. Flicek P, Amode MR, Barrell D, Beal K, Brent S, Carvalho-Silva D, Clapham P, Coates G, Fairley S, Fitzgerald S, Gil L, Gordon L, Hendrix M, Hourlier T, Johnson N, Kähäri AK, Keefe D, Keenan S, Kinsella R, Komorowska M, Koscielny G, Kulesha E, Larsson P, Longden I, McLaren W, Muffato M, Overduin B, Pignatelli M, Pritchard B, Riat HS, et al: Ensembl 2012. Nucleic Acids Res 2012, 40:D84-90.

52. Fujita PA, Rhead B, Zweig AS, Hinrichs AS, Karolchik D, Cline MS, Goldman M, Barber GP, Clawson H, Coelho A, Diekhans M, Dreszer TR, Giardine BM, Harte RA, Hillman-Jackson J, Hsu F, Kirkup V, Kuhn RM, Learned K, Li CH, Meyer LR, Pohl A, Raney BJ, Rosenbloom KR, Smith KE, Haussler D, Kent WJ: The UCSC Genome Browser database: update 2011. Nucleic Acids Res 2010, 39:D876-D882.

53. Ivics Z, Hackett PB, Plasterk RH, Izsvák Z: Molecular reconstruction of Sleeping Beauty, a Tc1-like transposon from fish, and its transposition in human cells. Cell 1997, 91:501-510.

54. Kent WJ: BLAT--The BLAST-Like Alignment Tool. Genome Res 2002, 12:656-664.

55. Kawakami K, Abe G, Asada T, Asakawa K, Fukuda R, Ito A, Lal P, Mouri N, Muto A, Suster ML, Takakubo H, Urasaki A, Wada H, Yoshida M: zTrap: zebrafish gene trap and enhancer trap database. BMC Dev Bio/ 2010 10:105.

56. Balciunas D, Davidson AE, Sivasubbu S, Hermanson SB, Welle Z, Ekker SC: Enhancer trapping in zebrafish using the Sleeping Beauty transposon. BMC Genomics 2004, 5:62

57. Choo BGH, Kondrichin I, Parinov S, Emelyanov A, Go W, Toh W-C, Korzh V: Zebrafish transgenic Enhancer TRAP line database (ZETRAP). BMC Dev Biol 2006, 6:5.

58. Ellingsen S, Laplante MA, König M, Kikuta H, Furmanek T, Hoivik EA, Becker TS: Large-scale enhancer detection in the zebrafish genome. Development 2005, 132:3799-3811.

59. Allen ND, Cran DG, Barton SC, Hettle S, Reik W, Surani MA: Transgenes as probes for active chromosomal domains in mouse development. Nature 1988, 333:852-855.

60. Gossler A, Joyner AL, Rossant J, Skarnes WC: Mouse embryonic stem cells and reporter constructs to detect developmentally regulated genes. Science 1989, 244:463-465

61. Ding S, Wu X, Li G, Han M, Zhuang Y, Xu T: Efficient transposition of the piggyBac (PB) transposon in mammalian cells and mice. Cell 2005, 122:473-483.

62. Horie K, Yusa K, Yae K, Odajima J, Fischer SEJ, Keng WW, Hayakawa T, Mizuno S, Kondoh G, ljiri T, Matsuda Y, Plasterk RHA, Takeda J: Characterization of Sleeping Beauty transposition and its application to genetic screening in mice. Mol Cell Biol 2003, 23:9189-9207.

63. Mátés L, Chuah MKL, Belay E, Jerchow B, Manoj N, Acosta-Sanchez A, Grzela DP, Schmitt A, Becker K, Matrai J, Ma L, Samara-Kuko E, Gysemans C, Pryputniewicz D, Miskey C, Fletcher B, Vandendriessche T, Ivics Z, Izsvák Z: Molecular evolution of a novel hyperactive Sleeping Beauty transposase enables robust stable gene transfer in vertebrates. Nat Genet 2009, 41:753-761.

64. Kelsch W, Stolfi A, Lois C: Genetic labeling of neuronal subsets through enhancer trapping in mice. PLoS One 2012, 7:e38593.

65. Pennacchio LA, Ahituv N, Moses AM, Prabhakar S, Nobrega MA, Shoukry M, Minovitsky S, Dubchak I, Holt A, Lewis KD, Plajzer-Frick I, Akiyama J, De Val S, Afzal V, Black BL, Couronne O, Eisen MB, Visel A, Rubin EM: In vivo enhancer analysis of human conserved non-coding sequences. Nature 2006, 444:499-502.

66. Kikuta $H$, Laplante $M$, Navratilova $P$, Komisarczuk AZ, Engström PG, Fredman D, Akalin A, Caccamo M, Sealy I, Howe K, Ghislain J, Pezeron G, Mourrain P, Ellingsen S, Oates AC, Thisse C, Thisse B, Foucher I, Adolf B, Geling A, Lenhard B, Becker TS: Genomic regulatory blocks encompass multiple neighboring genes and maintain conserved synteny in vertebrates. Genome Res 2007, 17:545-555. 
67. Engström $P G$, Fredman $D$, Lenhard B: Ancora: a web resource for exploring highly conserved noncoding elements and their association with developmental regulatory genes. Genome Biol 2008, 9:R34.

68. Dixon JR, Selvaraj S, Yue F, Kim A, Li Y, Shen Y, Hu M, Liu JS, Ren B: Topological domains in mammalian genomes identified by analysis of chromatin interactions. Nature 2012, 485:376-380.

69. Nora EP, Lajoie BR, Schulz EG, Giorgetti L, Okamoto I, Servant N, Piolot T, van Berkum NL, Meisig J, Sedat J, Gribnau J, Barillot E, Blüthgen N, Dekker J, Heard E: Spatial partitioning of the regulatory landscape of the X-inactivation centre. Nature 2012, 485:381-385.

70. Shen Y, Yue F, McCleary DF, Ye Z, Edsall L, Kuan S, Wagner U, Dixon J, Lee L, Lobanenkov W, Ren B: A map of the cis-regulatory sequences in the mouse genome. Nature 2012, 488:116-120.

71. Kim E, Kim S, Kim DH, Choi B-S, Choi I-Y, Kim J-S: Precision genome engineering with programmable DNA-nicking enzymes. Genome Res 2012, 22:1327-1333.

72. Wang J, Friedman G, Doyon Y, Wang NS, Li CJ, Miller JC, Hua KL, Yan JJ, Babiarz JE, Gregory PD, Holmes MC: Targeted gene addition to a predetermined site in the human genome using a ZFN-based nicking enzyme. Genome Res 2012, 22:1316-1326.

doi:10.1186/1471-2164-14-215

Cite this article as: Chen et al.: TRACER: a resource to study the regulatory architecture of the mouse genome. BMC Genomics 2013 14:215

\section{Submit your next manuscript to BioMed Central and take full advantage of:}

- Convenient online submission

- Thorough peer review

- No space constraints or color figure charges

- Immediate publication on acceptance

- Inclusion in PubMed, CAS, Scopus and Google Scholar

- Research which is freely available for redistribution 